\title{
Sarcoma indiferenciado (embrionário) do fígado: relato de caso e revisão da literatura
}

\section{Undifferentiated (embryonal) sarcoma of the liver: a case report and review of the literature}

Vanderlei Segatelli( ${ }^{(1)}$; Ebe Christie de Oliveira(1); Syomara Pereira da Costa Melo(2), Gustavo Neves( ${ }^{(3)}$; Carlos Eduardo Bacchi(4); Cecília Amélia Fazzio Escanhoela(5)

\begin{tabular}{l}
\multicolumn{1}{c|}{ unitermos } \\
Sarcoma \\
Tumores \\
Fígado
\end{tabular}

\section{resumo}

O sarcoma embrionário (indiferenciado) é uma neoplasia mesenquimal maligna incomum e primária do fígado, que acomete principalmente crianças na faixa etária entre 6 e 10 anos de idade. Este trabalho tem como objetivo relatar um caso dessa neoplasia, enfatizando os aspectos clínicos e anatomopatológicos, bem como revisar a literatura sobre o tema.

\section{Introdução}

O sarcoma embrionário (indiferenciado) do fígado é uma neoplasia maligna rara de etiopatogenia ainda desconhecida, que acomete a população pediátrica, principalmente na faixa etária entre 6 e 10 anos de idade, sem predileção por sexo, e raramente ocorre em adultos ${ }^{(4,5,9)}$.

Clinicamente, a maioria dos casos apresenta expansão abdominal, com tumor palpável doloroso na região epigástrica ou no hipocôndrio direito, de rápido crescimento. Alguns pacientes podem apresentar sintomas gastrintestinais inespecíficos e perda de peso. As provas de função hepática geralmente apresentam níveis dentro dos padrões de normalidade. Em alguns casos há relato de aumento da fosfatase alcalina sérica ${ }^{(6)}$.

Os aspectos radiológicos encontrados na ultrasonografia e na tomografia computadorizada variam entre imagens sólidas e císticas ${ }^{(12,13,17)}$. Os tumores com importante componente cístico podem simular hamartoma mesenquimal e cistos hidáticos do fígado( ${ }^{(8,18)}$.

\section{Relato de caso}

Paciente, sexo feminino, 5 anos, proveniente do Nordeste brasileiro, procurou atendimento médico com

1. Médicos patologistas do Laboratório de Patologia e Citologia de Sorocaba (SP).

2. Médica patologista do Departamento de Patologia do Hospital Universitário (Unidade Presidente Dutra) da Universidade Federal do Maranhão (HU/UFM)

3. Médico oncologista do Hospital do Câncer Infantil Sarina Rolim Caracante de Sorocaba (SP).

4. Médico patologista; diretor responsável da Consultoria em Patologia de Botucatu (SP).

5. Professora-doutora do Departamento de Anatomia Patológica da Faculdade de Ciências Médicas da Universidade Estadual de Campinas (FCM/UNICAMP)

Caso apresentado resumidamente como pôster no XXV Congresso Brasileiro de Patologia realizado em Natal (2005). 
histórico de dor abdominal há 15 dias. Ao exame físico, apresentava-se em bom estado geral, observando-se tumor palpável na região epigástrica.

Os exames de ultra-sonografia e tomografia computadorizada abdominais mostraram imagem heterogênea, sólido-cística, de contornos irregulares, localizada no lobo esquerdo do fígado. A paciente foi submetida à hepatectomia parcial com exérese completa do tumor.

Ao exame anatomopatológico observou-se, no estudo macroscópico, neoplasia de limites irregulares $(10 \times 8 \times 7 \mathrm{~cm})$, com áreas sólidas pardo-vinhosas, bem como múltiplas áreas císticas e hemorrágicas. Microscopicamente, os cortes histológicos corados com hematoxilina e eosina (HE) demonstraram neoplasia maligna composta por células com núcleos fusiformes e pleomórficos, bizarros, algumas multinucleadas, dispersas num estroma ora fibroso ora mixóide, rico em mucopolissacarídeos e com ductos biliares isolados de vários tamanhos (Figuras 1A e 1B).

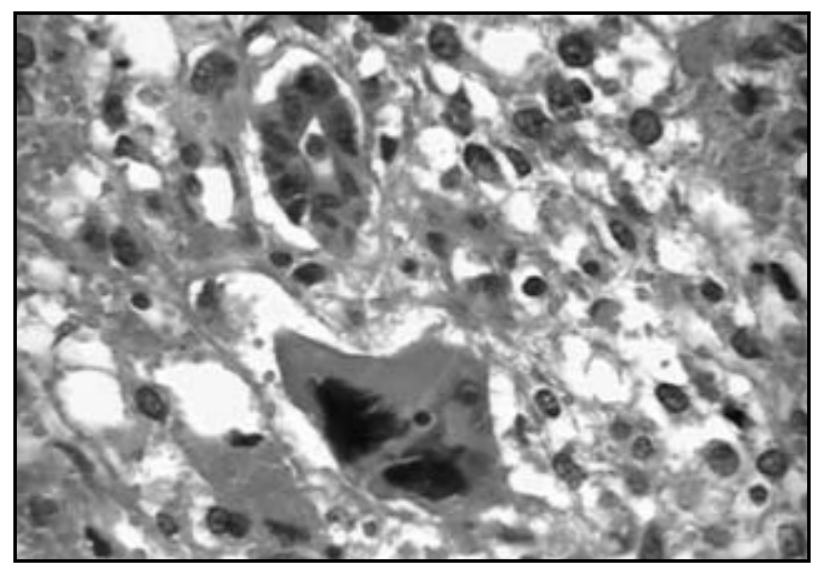

Figura 1A - Células neoplásicas com núcleos pleomórficos e ducto biliar isolado dispersos em estroma mixóide (hematoxilina-eosina - 40x)

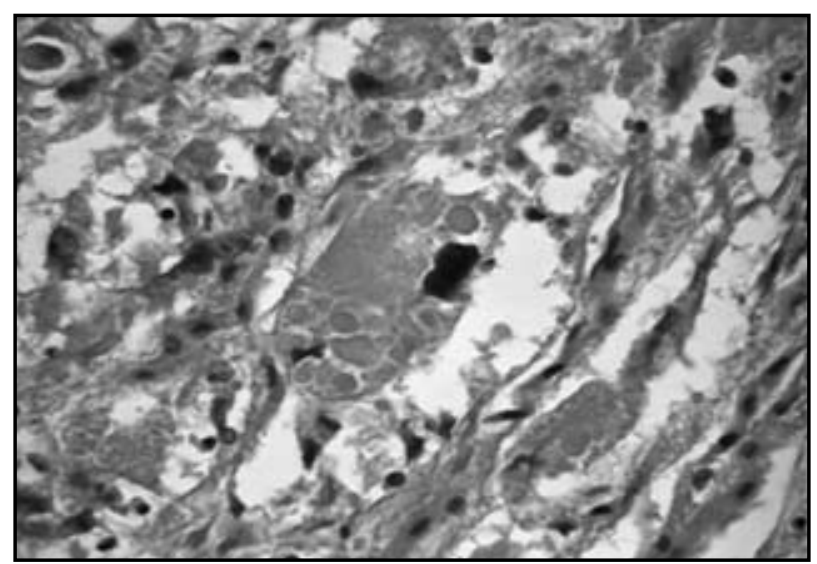

Figura $1 B$ - Células neoplásicas apresentando glóbulos hialinos intracitoplasmáticos (hematoxilina-eosina-40x)
As células neoplásicas apresentam como característica importante a presença de glóbulos hialinos intracitoplasmáticos, positivos na coloração histoquímica do ácido periódico de Schiff (PAS) com diástase (Figura 2).

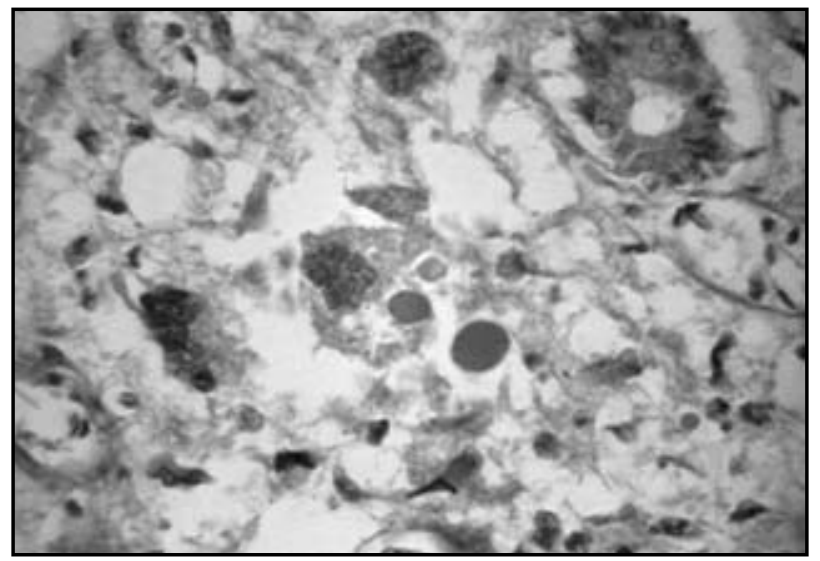

Figura 2 - Células neoplásicas apresentando núcleos pleomórficos e glóbulos intracitoplasmáticos PAS diastase-resistentes (PAS com diastase - 40X)

O estudo imuno-histoquímico demonstrou positividade nas células neoplásicas para desmina (D33) e $\alpha$-1-antitripsina (policlonal). Portanto, os estudos morfológico, histoquímico e imuno-histoquímico definiram o diagnóstico de sarcoma embrionário (indiferenciado) do fígado.

Foram realizados três ciclos de quimioterapia (protocolo VAIA-CWS: vincristina, actinomicina $D$, ifosfamida e doxorrubicina). Atualmente, dois anos após o diagnóstico e tratamento, a paciente apresenta-se em bom estado geral e sem sinais de recorrência da neoplasia.

\section{Discussão}

O sarcoma embrionário (indiferenciado) foi primeiramente descrito como uma entidade clinicopatológica distinta por Stocker et al. ${ }^{(20)}$, em 1978, com a publicação de uma série de 31 casos e, até hoje, ainda não possui uma etiopatogenia definida.

Estudos ultra-estruturais e imuno-histoquímicos indicam origem mesenquimal do tumor, porém não há definição quanto a uma linhagem celular específica. Através de trabalhos que demonstraram a sobreposição de características imunohistoquímicas e ultra-estruturais semelhantes, alguns autores sugerem histogênese comum entre o sarcoma indiferenciado e o rabdomiossarcoma do fígado, provavelmente decorrente de uma célula-tronco mesenquimal multipotencial $(9,14,16)$.

Algumas evidências sugerem que o sarcoma embrionário (indiferenciado) do fígado seja derivado, em alguns 
casos, da transformação maligna do hamartoma mesenqui$\mathrm{mal}^{(1,10,15,21)}$. Estudos genéticos reascendem a idéia de uma histogênese comum entre essas duas neoplasias ${ }^{(3)}$.

Na literatura são descritas algumas variedades de alterações cromossômicas envolvidas na patogênese da neoplasia, mas ainda não há uma anormalidade genética específica ${ }^{(19)}$. Estudos recentes enfatizam a possibilidade do envolvimento de genes específicos, como o Tp53, no desenvolvimento do sarcoma embrionário(11).
O sarcoma embrionário é considerado uma neoplasia agressiva e de prognóstico desfavorável, com sobrevida de 1 a 2 anos. Nos casos em que há predomínio de padrão cístico, a ruptura da neoplasia é uma complicação importante ${ }^{(7,22)}$.

Nas últimas décadas, o prognóstico dos pacientes com sarcoma indiferenciado do fígado melhorou expressivamente, com a associação da ressecção cirúrgica completa do tumor e tratamentos adjuvantes, principalmente novos protocolos quimioterápicos ${ }^{(2,23,24)}$.

\section{Referências}

1. BEGUERET, H. et al. Hepatic undifferentiated embryonal sarcoma: malignant evolution of mesenchymal hamartoma? Study of one case with immunohistochemical and flow cytometric emphasis. J Hepatol, v. 34, n. 1, p. 178-9, 2001.

2. BISOGNO, G. et al. Undifferentiated sarcoma of the liver in childhood: a curable disease. Cancer, v. 94, n. 1, p. 252-7, 2002.

3. BOVE, K.E.; BLOUGH, R.I.; SOUKUP, S. Third report of $\mathrm{t}(19 q)$ (13.4) in mesenchymal hamartoma of liver with comments on link to embryonal sarcoma. Pediatr Dev Pathol, v. 1, p. 438-42, 1998.

4. DAI, C.L. et al. Undifferentiated (embryonal) sarcoma of liver in adult: a case report. World J Gastroenterol, v. 11, n. 6, p. 926-9, 2005.

5. DIEDHIOU, A. et al. Undifferentiated embryonal sarcoma of the liver in an adult: a case report. Ann Pathol, v. 22, n. 2, p. 134-6, 2002.

6. HAMILTON, S.R. et al. Pathology \& Genetics - Tumours of the digestive system. In: HAMILTON, S.R.; AALTONEN, L.A. (eds.). World Health Organization Classification of Tumours. Lyon: IARC Press, 2000. p. 194-5.

7. HUNG, T.Y.; LU, D.; LIU, M.C. Undifferentiated (embryonal) sarcoma of the liver complicated with rupture in a child. J Pediatr Hematol Oncol, v. 29, n. 1, p. 63-5, 2007.

8. JOSHI, S.W.; MERCHANT, N.H.; JAMBHEKAR, N.A. Primary multilocular cystic undifferentiated (embryonal) sarcoma of the liver in childhood resembling hydatid cyst of the liver. Br J Radiol, v. 70, p. 314-6, 1997.

9. LACK, E.E. et al. Undifferentiated (embryonal) sarcoma of the liver: clinical and pathologic study of 16 cases with emphasis on immunohistochemical features. Am J Surg Pathol, v. 15, n. 1. p. 1-16, 1991.

10. LAUWERS, G.Y. et al. Hepatic undifferentiated (embryonal) sarcoma arising in a mesenchymal hamartoma. Am J Surg Pathol, v. 21, n. 10, p. 1248-254, 1997.

11. LEPREUX, S. et al. Mutation of TP53 gene is involved in carcinogenesis of hepatic undifferentiated (embryonal) sarcoma of the adult, in contrast with Wnt or telomerase pathways: an immunohistochemical study of three cases with genomic relation in two cases. J Hepatol, v. 42, n. 3, p. 424-9, 2005.

12. MOON, W.K. et al. Undifferentiated embryonal sarcoma of the liver: US and CT findings. Pediatr Radiol, v. 24, n. 7, p. 500-3, 1994.

13. NETO, S.A.A.; SOUZA, A.S. Sarcoma embrionário indiferenciado do fígado: relato de caso. Radiol Bras, v. 34, n. 5, p. 305-8, 2001.

14. NISHIO, J. et al. Undifferentiated (embryonal) sarcoma of the liver in middle-aged adults: smooth muscle differentiation determined by immunohistochemistry and electron microscopy. Hum Pathol, v. 34, n. 3, p. 246-52, 2003.

15. O'SULLIVAN, M.J. et al. Undifferentiated embryonal sarcoma with unusual features arising within mesenchymal hamartoma of the liver: report of a case and review of the literature. Pediatr Dev Pathol, v. 4 n. 5, p. 482-9, 2001.

16. PARHAM, D.M. et al. Immunohistochemical and ultrastructural spedtrum of hepatic sarcomas of childhood: evidence for a common histogenesis. Mod Pathol, v. 4, n. 5, p. 648-53, 1991.

17. ROS, P.R. et al. Undifferentiated (embryonal) sarcoma of the liver: radiologic-pathologic correlation. Radiology, v. 161, n. 1, p. 141-5, 1986.

18. SHAH, S.R. et al. Cystic variant of embryonal sarcoma of liver. Indian J Gastroenterol, v. 21, n. 1, p. 35-6, 2002.

19. SOWERY, R.D. et al. Comparative genomic hybridization detects multiple chromosomal amplifications and deletions in undifferentiated embryonal sarcoma of the liver. Cancer Genet Cytogenet, v. 126, n. 2, p 128-33, 2001.

20. STOCKER, J.T.; ISHAK, K.G. Undifferentiated (embryonal) sarcoma of the liver. Cancer, v. 42, p. 336-48, 1978.

21. STRINGER, M.D.; ALIZAI, N.K. Mesenchymal hamartoma of the liver: a systematic review. J Pediatr Surg, v. 40, n. 11, p. 1681-690, 2005

22. UCHIYAMA, M. et al. Treatment of ruptured undifferentiated sarcoma of the liver in children: a report of two cases and review of the literature. J Hepatobiliary Pancreat Surg, v. 8, n. 1, p. 87-91, 2001.

23. URBAN, C.E. etal. Undifferentiated (embryonal) sarcoma of the liver in childhood: successful combined-modality therapy in four patients. Cancer, v. 72, n. 8, p. 2511-6, 1993.

24. WEBBER, E.M. et al. Undifferentiated embryonal sarcoma of the liver: results of clinical management in one center. J Pediatr Surg, v. 34, n. 11, p. 1641-44, 1999. 